\title{
Groups that are pairwise nilpotent
}

\author{
Gérard Endimioni \\ C.M.I, Université de Provence, UMR-CNRS 6632 \\ 39, rue F. Joliot-Curie, 13453 Marseille Cedex 13, France \\ E-mail: endimion@gyptis.univ-mrs.fr \\ Gunnar Traustason \\ Department of Mathematical Sciences, University of Bath, \\ Bath BA2 7AY, UK \\ E-mail: gt223@maths.bath.ac.uk
}

\begin{abstract}
In this paper we study groups generated by a set $X$ with the property that every two elements in $X$ generate a nilpotent subgroup.
\end{abstract}

\section{Introduction}

In this paper we deal with groups that have a special type of presentation.

Definition 1 Let $G$ be a group with a generating set $X$. We say that $(G, X)$ is a pairwise nilpotent presentation of $G$ if one has the property that any pair of elements $x, y \in X$ generates a nilpotent subgroup. A group that has a pairwise nilpotent presentation will be referred to as a pairwise nilpotent group.

Of course every locally nilpotent group is pairwise nilpotent and more generally every group with the property that all 2-generator subgroups are nilpotent. Another example of a pairwise nilpotent group is any 2-group generated 
by involutions. In fact if $G$ is a 2-group and $a_{1}, a_{2} \in G$ are distinct involutions with $a_{1} a_{2}$ of order $2^{n}$ then $\left\langle a_{1}, a_{2}\right\rangle$ is the dihedral group of order $2^{n+1}$.

Definition 2 Let $(G, X)$ be a pairwise nilpotent presentation. Let $N$ be a function that assigns to every pair of distinct elements $x, y \in X$ a positive integer $N(x, y)$. If $\langle x, y\rangle$ is nilpotent of class at most $N(x, y)$, we say that the presentation is of type $N$.

We will in particular focus on presentations where the number of generators is three. Suppose $X=\langle a, b, c\rangle$ and that $\langle a, b\rangle$ is nilpotent of class $r,\langle a, c\rangle$ of class $s$ and $\langle b, c\rangle$ of class $t$. In this case we will usually say that the presentation is of type $(r, s, t)$. We will say a group $G$ is of type $(r, s, t)$ if it has a presentation of type $(r, s, t)$.

We are interested in finding criteria under which a finitely generated pairwise nilpotent group must be nilpotent. All groups of exponent 4 are known to be locally finite. As we mentioned above every 2-group generated by two involutions is finite. It is still an open question whether a group of exponent 8 generated by three involutions must be finite. The best result here seems to be one of Hermanns [5] who has shown that every group of exponent 8 generated by three involutions and of type $(1,2,3)$ must be nilpotent.

Another motivation for our study comes from the work on 4-Engel groups. It is a long-standing conjecture that an $n$-Engel group is locally nilpotent. In [4] the affirmative answer is given for $n=4$. In proving that 4-Engel groups are locally nilpotent an important step was to show that any 4-Engel group of type $(1,2,3)$ is nilpotent $[4,7]$. In fact any group $\langle a, b, c\rangle$ of type $(1,2,3)$ with the extra properties that $[b, c, c]=1$ and that the generators are of $\{2,3\}$-free order must be nilpotent [7].

By a result of Zorn [8], every finite group with all two generator subgroups nilpotent must be nilpotent. Hence if $(G, G)$ is a pairwise nilpotent presentation of a finite group then $G$ is nilpotent. This property fails miserably for infinite groups as there are famous non-nilpotent three generator examples of Golod [1] for which every two generator subgroup is nilpotent.

Let us consider for the moment groups with a three generator pairwise nilpotent presentation. Of course any group $\langle a, b, c\rangle$ with a pairwise nilpotent presentation of type $(1,1, t)$ must be nilpotent as $a$ is in the center. It is 
hardly surprising that apart from these obvious cases we can not deduce global nilpotence. In fact in almost all the situations we can not even say that the group is solvable. There is one interesting exception: in Section 2 we will see that any group of type $(1,2,2)$ is solvable of derived length at most 3 and if the generators are of 2 -free order the group is furthermore nilpotent. However we will see in Section 3 that there are non-solvable groups of types $(1,2,3)$ and $(2,2,2)$.

In Section 4 we deal with pairwise nilpotent groups that are solvable. Here we have some positive results. We observe that any pairwise nilpotent group that is nilpotent-by-abelian must be nilpotent. This is not true for solvable groups in general and there are already pairwise nilpotent groups that are abelian-by-(class 2) but that are non-nilpotent. However if all the generators are of $\{2,3\}$-free order the group must be nilpotent. We make the following conjecture:

Conjecture 1. For each positive integer $c$ there exists a finite set of primes $\mathcal{P}_{c}$ so that any pairwise nilpotent group that is abelian-by-(class $\left.c\right)$ generated by elements of $\mathcal{P}_{c}$-free order must be nilpotent.

If this conjecture is true it would follow in particular that any solvable torsionfree group that is pairwise nilpotent group must be nilpotent. In Section 4 we also study the minimal finite solvable groups that are pairwise nilpotent but non-nilpotent. These are minimal in the sense that any pairwise nilpotent proper subgroup and quotient is nilpotent. We also give examples that give lower bounds for the largest prime in $\mathcal{P}_{c}$, provided that this set of exceptional primes is finite.

In Section 5 we consider bounds for the class. We will see that any nilpotent group of type $(1,2,2)$ with generators of 2 -free order, is nilpotent of class at most 3 and that any nilpotent group of type $(1,2,3)$ with generators of $\{2,3\}$-free order is nilpotent of class at most 5 . We will also see that for any prime $p$ there are nilpotent $p$-groups of types $(2,2,2)$ and $(1,3,3)$ of arbitrary large class. Of course any group of type $(1,1, t)$ is nilpotent of class at most $t$. This leaves out groups of type $(1,2, t)$ where $t \geq 4$. We make the following conjecture.

Conjecture 2. For each positive integer $t$ there exists a finite set of primes 
$\mathcal{P}_{t}$ and a positive integer $c(t)$ such that any nilpotent group of type $(1,2, t)$ with generators of $\mathcal{P}_{t}$-free order is nilpotent of class at most $c(t)$.

In this paper we will be using the left normed convention for commutators. That is: $[x, y]=x^{-1} y^{-1} x y$ and $[x, y, z]=[[x, y], z]$.

\section{Groups of type $(1,2,2)$}

We start with a generic example.

Example. Let

$$
D_{n}=\left\langle a, b: a^{2}=b^{2}=1,(a b)^{n}=1\right\rangle
$$

be the dihedral group of order $2 n$ ( $n$ here possibly $\infty$ ). Let

$$
E_{n}=D_{n} \operatorname{wr}\langle c\rangle
$$

where $c$ is an involution.

Lemma 1 If $n$ is coprime to 2 then $E_{n}(a, b, c)=\left\langle a, b^{c}, c\right\rangle$ is a non-nilpotent group of type $(1,2,2)$.

Proof To see this notice first that $a, b^{c}$ obviously commute. Then

$$
[c, a, a]=\left[a^{c} a, a\right]=1
$$

and

$$
[a, c, c]=\left[a a^{c}, c\right]=a^{c} a a^{c} a=1
$$

which shows that $\langle a, c\rangle$ is nilpotent of class 2 . Similarly we see that $\left\langle b^{c}, c\right\rangle$ is nilpotent of class 2 .

The next theorem shows that, in a sense, these examples generate all nonnilpotent $(1,2,2)$ groups.

Theorem 1 Let $G=\langle a, b, c\rangle$ be of type (1,2,2). Then $G$ is soluble of derived length at most three, and has a finitely generated nilpotent normal subgroup $N$ such that

$$
G / N=\langle\bar{a}, \bar{b}, \bar{c}\rangle
$$

is a quotient of $E_{n}\left(a, b^{c}, c\right)$ for some $n$. In particular we have that $a^{2}, b^{2}, c^{2} \in$ $N$. 
Proof As $\langle c\rangle^{\langle a\rangle}$ is abelian and $1=[c, a, a]$ we have

$$
c^{a^{2}}=c^{2 a} c^{-1} \text {. }
$$

Using $\left[c, c^{b}\right]=1$, it follows that

$$
\begin{aligned}
1 & =\left[c^{a^{2} b}, c^{a^{2}}\right] \\
& =\left[c^{2 a b} c^{-b}, c^{2 a} c^{-1}\right] \\
& =\left[c^{2 a b}, c^{2 a} c^{-1}\right] c^{-b}\left[c^{-b}, c^{2 a} c^{-1}\right] \\
& =\left[c^{2 a b}, c^{-1}\right]^{-b}\left[c^{-b}, c^{2 a}\right]^{c^{-1}} \\
& =\left[c^{2 a b}, c^{-1}\right]\left[c^{-b}, c^{2 a}\right]
\end{aligned}
$$

and hence

$$
\left[c^{-1}, c^{2 a b}\right]=\left[c^{-b}, c^{2 a}\right] .
$$

The RHS commutes with $c^{a}$ and $c^{b}$, while the LHS commutes with $c$ and $c^{a b}$. Thus, both sides are in the centre of

$$
\langle c\rangle^{G}=\left\langle c, c^{a}, c^{b}, c^{a b}\right\rangle .
$$

Consider the group

$$
M=\left\langle c^{2}\right\rangle^{G}=\left\langle c^{2}, c^{2 a}, c^{2 b}, c^{2 a b}\right\rangle
$$

The only non-trivial commutators in these generators are $\left[c^{2}, c^{2 a b}\right]$ and $\left[c^{2 b}, c^{2 a}\right]$ which as we have seen are in the centre of $\langle c\rangle^{G}$. In particular we have that $M$ is nilpotent of class at most 2 . Now consider

$$
N=\left\langle a^{2}, b^{2}, c^{2}\right\rangle^{G}
$$

As

$$
\left[a^{2}, c\right]=[a, c]^{2}=\left[a, c^{2}\right] \in M
$$

and

$$
\left[b^{2}, c\right]=[b, c]^{2}=\left[b, c^{2}\right] \in M
$$

it follows that

$$
N=\left\langle a^{2}, b^{2}\right\rangle M
$$

As $\left\langle c^{2}, a^{2}\right\rangle,\left\langle c^{2}, a^{2}\right\rangle^{a},\left\langle c^{2}, a^{2}\right\rangle^{b},\left\langle c^{2}, b^{2}\right\rangle,\left\langle c^{2}, b^{2}\right\rangle^{a},\left\langle c^{2}, b^{2}\right\rangle^{b}$ are all nilpotent of class at most 2 , and $a^{2}$ commutes to $b^{2}$, we have

$$
\left[M,_{3} N\right] \leq \gamma_{2}(M)
$$


Then

$$
\left[M, M,_{5} N\right] \leq \prod_{i+j=5}\left[\left[M_{i} N\right],\left[M,{ }_{j} N\right]\right] \leq \gamma_{3}(M)=1
$$

As $N^{\prime} \leq M$ we thus have $\gamma_{10}(N)=1$.

Now consider

$$
G / N=\langle\bar{a}, \bar{b}, \bar{c}\rangle .
$$

Then $\bar{a}, \bar{b}, \bar{c}$ are involutions. Let $D=\left\langle\bar{a}, \bar{b}^{c}\right\rangle$ then $D^{c}=\left\langle\bar{a}^{c}, \bar{b}\right\rangle$. Notice that $\left[D, D^{c}\right]=1$ and that $D$ is dihedral. Also

$$
G / N=\left(D D^{c}\right) \cdot\langle\bar{c}\rangle
$$

which is clearly a quotient of some $E_{n}\left(\bar{a}, \bar{b}^{c}, \bar{c}\right)$.

It remains to show that $G^{(3)}=\{1\}$. As a preparation we first make a useful observation. We have seen that $\left[c^{-b}, c^{2 a}\right]=\left[c^{-1}, c^{2 a b}\right]$ is in $R=Z\left(\langle c\rangle^{G}\right)$. As $c^{2 a}$ commutes with $c$ and $c^{a b}$ it follows that $c^{2 a} \in Z^{2}\left(\langle c\rangle^{G}\right)$. But $\left.Z^{2}(\langle c\rangle)^{G}\right)$ is normal in $G$ and hence $\left\langle c^{2}\right\rangle^{G} \leq Z^{2}\left(\langle c\rangle^{G}\right)$. Let us now turn back to the derived length of $G$. First notice that $G^{\prime} \leq\langle c\rangle^{G}=\left\langle c, c^{a}, c^{b}, c^{a b}\right\rangle$. The only nontrivial commutators in these generators are $\left[c^{a}, c^{b}\right]$ and $\left[c, c^{a b}\right]$ and thus $G^{\prime \prime} \leq\left\langle\left[c^{a}, c^{b}\right],\left[c, c^{a b}\right]\right\rangle^{G}$. As $c^{a}, c^{b}$ commute with $c, c^{a b}$ it suffices to show that

$$
G^{\prime \prime} \leq\left\langle\left[c^{a}, c^{b}\right],\left[c, c^{a b}\right]\right\rangle R
$$

It now remains to see that $\left[c^{a}, c^{b}\right]^{x}$ and $\left[c, c^{a b}\right]^{x}$ are in $\left\langle\left[c^{a}, c^{b}\right]\right\rangle R$ for $x \in$ $\left\langle a, a^{-1}, b, b^{-1}, c, c^{-1}\right\rangle$. But these come from straightforward calculations using $c^{a^{2}}=c^{2 a} c^{-1}, c^{a^{-1}}=c^{2} c^{-a}, c^{b^{2}}=c^{2 b} c^{-1}, c^{b^{-1}}=c^{2} c^{-b}$ and $\left\langle c^{2}\right\rangle^{G} \leq Z^{2}\left(\langle c\rangle^{G}\right)$.

For example we have modulo $R$

$$
\left[c^{a}, c^{b}\right]^{a} \equiv\left[c^{2 a} c^{-2} c, c^{a b}\right] \equiv\left[c, c^{a b}\right]
$$

This finishes the proof.

Remark If $G$ is generated by torsion elements $a, b, c$ of 2-prime order then $G=N$ as $a^{2}, b^{2}, c^{2} \in N$. Thus $G$ is nilpotent and in section 5 we will see that the class is at most 3 . If $G$ is a 2 -group however then the class is unbounded as $E_{2^{n}}(a, b, c)$ has class at least $n$. 


\section{The question of solubility}

Whereas all groups of type $(1,2,2)$ are soluble of derived length at most three we will see in this section that there are non-soluble groups of types $(1,2,3)$ and $(2,2,2)$.

All the examples will be obtained through different pairwise nilpotent presentations of the group

$$
E=A_{5} \operatorname{wr} C_{3}
$$

Lemma 2 If $\left\{i_{1}, i_{2}, i_{3}\right\},\left\{j_{1}, j_{2}, j_{3}\right\}$ are two three-element sets with union $\{1,2,3,4,5\}$ then

$$
A_{5}=\left\langle\left(i_{1}, i_{2}, i_{3}\right),\left(j_{1}, j_{2}, j_{3}\right)\right\rangle .
$$

Proof Without loss of generality we can suppose that $i_{1}=1, i_{2}=2, i_{3}=$ $3, j_{1}=1, j_{2}=4, j_{3}=5$. Let $a=(1,2,3), b=(1,4,5)$, and $H=\langle a, b\rangle$. We want to show that $H=A_{5}$. Notice first that $H$ contains $c=a b=(1,2,3,4,5)$ and thus 5 divides $|H|$. As $(1,2,3) \in H$ we also have that 3 divides $|H|$. Then $H$ contains

$$
a^{c}=(1,2,3)^{(1,2,3,4,5)}=(2,3,4)
$$

and then also

$$
\begin{aligned}
a a^{c} & =(1,2,3)(2,3,4)=(1,3)(2,4) \\
\left(a a^{c}\right)^{a} & =[(1,3)(2,4)]^{(1,2,3)}=(2,1)(3,4) .
\end{aligned}
$$

That is $H$ contains the Klein- 4 group and 4 divides $|H|$. From this it follows that 60 divides $|H|$ and thus $H=A_{5}$.

Lemma 3 Let $a=\left(i_{1}, i_{2}, i_{3}\right)$ and $b=\left(j_{1}, j_{2}, j_{3}\right)$ be as in the previous lemma. Then $E$ is of type $(1,3,3)$.

Proof Suppose that $C_{3}=\langle c\rangle$ and that $a, b$ are as in previous lemma. By that lemma we have that

$$
E=\left\langle a, b^{c}, c\right\rangle .
$$

Clearly $a$ and $b^{c}$ commute. As $\langle a\rangle^{\langle c\rangle}$ is abelian, any commutator in $a, c$ that involves $a$ twice is trivial. As

$$
[a, c, c, c]=a^{(-1+c)^{3}}=a^{-1+c^{3}}=a^{0}=1
$$


it follows that $\langle a, c\rangle$ is nilpotent of class at most 3. Similarly $\left\langle b^{c}, c\right\rangle$ is nilpotent of class at most 3 .

Thus $E$ is a non-soluble group of type $(1,3,3)$.

Lemma 4 Let $a, b$, and $c$ be as in the previous lemma and its proof. If $F=\left\langle\left[a, c^{2}\right], b^{c}, c\right\rangle$, then $F=E$ and the presentation is of type $(1,2,3)$.

Proof Clearly $\left[a, c^{2}\right]=a^{-1} a^{c^{2}}$ commutes with $b^{c}$. We have already seen that $\left\langle b^{c}, c\right\rangle$ is nilpotent of class at most 3. Finally $\left\langle\left[a, c^{2}\right]\right\rangle^{\langle c\rangle}$ is abelian and thus any commutator in $\left[a, c^{2}\right]$ and $c$ that involves $\left[a, c^{2}\right]$ twice is trivial. As

$$
\left[\left[a, c^{2}\right], c, c\right]=a^{\left(-1+c^{2}\right)(-1+c)^{2}}=a^{(1+c)(-1+c)^{3}}=a^{0}=1
$$

it follows that $\left\langle\left[a, c^{2}\right], c\right\rangle$ is nilpotent of class at most 2. Thus $F$ is of type $(1,2,3)$. We show that $F=E$ by showing that $F$ contains $A_{5}$.

Firstly $F$ contains $b=(1,4,5)$ and thus

$$
b^{\left[a, c^{2}\right]}=b^{a^{-1} a^{c^{2}}}=b^{a^{-1}}=(1,4,5)^{(1,3,2)}=(3,4,5)
$$

and

$$
b^{\left[a, c^{2}\right]^{c^{-2}}}=b^{a^{-c^{-2} a}}=b^{a}=(1,4,5)^{(1,2,3)}=(2,4,5) .
$$

It follows that $F$ contains $(4,1,5)(4,5,2)=(4,1,2)$. By Lemma 2 we have that $F$ contains $\langle(4,1,2),(3,4,5)\rangle=A_{5}$.

Thus $E$ is an example of a non-soluble group of type $(1,2,3)$. We next show that $E$ is also of type $(2,2,2)$. First we remark that if $G=\langle a, b, c\rangle$ is any group of type $(1,2,3)$ then

$$
\tilde{G}=\left\langle a, c^{b}, c\right\rangle
$$

is of type $(2,2,2)$. This is because $\langle a, c\rangle$ is nilpotent of class 2 and thus also $\left\langle a, c^{b}\right\rangle=\left\langle a^{b}, c^{b}\right\rangle$. Furthermore, as $\langle b, c\rangle$ is nilpotent of class at most 3, we have that

$$
\left\langle c^{b}, c\right\rangle=\langle[c, b], c\rangle
$$

is nilpotent of class at most 2 .

Since $E=F=\left\langle\left[a, c^{2}\right], b^{c}, c\right\rangle$ is of type $(1,2,3)$, we have that $G=\left\langle\left[a, c^{2}\right], c^{b^{c}}, c\right\rangle$ is of type $(2,2,2)$. By the following Lemma, $E=G$ and this gives an example of a non-soluble group of type $(2,2,2)$. 
Lemma $5 G=E$.

Proof Notice first that

$$
\begin{aligned}
G & =\left\langle\left[a, c^{2}\right],\left[b^{c}, c\right], c\right\rangle \\
& =\left\langle a^{-1} a^{c^{2}}, b^{-c} b^{c^{2}}, c\right\rangle .
\end{aligned}
$$

The commutator of the first two elements is $[a, b]^{c^{2}}$ and as $G$ contains $c, G$ then contains

$$
[a, b]=(1,3,2)(1,2,3)^{(1,4,5)}=(1,3,2)(4,2,3)=(1,4,2)
$$

and then also

$$
[a, b]^{a^{-1} a^{c^{2}}}=[a, b]^{a^{-1}}=(1,4,2)^{(1,3,2)}=(3,4,1)
$$

and

$$
[a, b]^{\left(b^{-c} b^{c^{2}}\right)^{c^{-2}}}=[a, b]^{b}=(1,4,2)^{(1,4,5)}=(4,5,2) .
$$

By Lemma 2 we have that $A_{5}=\langle(3,4,1),(4,5,2)\rangle$.

\section{The question of nilpotence for solvable groups}

In this section we are interested in criteria for a solvable group that is pairwise nilpotent to be nilpotent. A starting point for our investigation is the following elementary fact.

Lemma 6 Let $G=\left\langle a_{1}, a_{2}, \ldots, a_{r}\right\rangle$ be a metabelian group for which every pair $a_{i}, a_{j}$ generates a nilpotent subgroup. Then $G$ is nilpotent.

Proof This follows easily from the following laws that hold in any metabelian group:

$$
\begin{gathered}
{[x, y, z][y, z, x][z, x, y]=1,} \\
{[u, x, y]=[u, y, x] \text { for any } u \in[G, G] .}
\end{gathered}
$$

Let us see why this is the case. For each $i \in\{1, \ldots, r\}$, let $n_{i}$ be the smallest non-negative integer such that $\left[x_{j, n_{i}+1} x_{i}\right]=1$ for all $1 \leq j \leq r$. We show that $G$ is nilpotent of class at most $n=n_{1}+n_{2}+\cdots+n_{r}$. In fact if we take a commutator of weight $n+1$ in the generators then some generator $x_{i}$ must 
occur at least $n_{i}+1$ times. Using the identities above, such a commutator is generated by commutators of the form

$$
\left[x_{j_{1}, n_{i}+1} x_{i}, x_{j_{2}}, \ldots, x_{j_{l}}\right]=1
$$

Hence $G$ is nilpotent of class at most $n$.

In fact using a well known nilpotence criterion of P. Hall we get the following generalisation.

Theorem 2 Let $G=\left\langle a_{1}, a_{2}, \ldots, a_{r}\right\rangle$ be a group that is nilpotent by abelian and for which every pair $a_{i}, a_{j}$ generates a nilpotent subgroup. Then $G$ is nilpotent.

Proof. Let $N$ be a normal nilpotent subgroup of $G$ such that $G / N$ is abelian. By last lemma $G /[N, N]$ is nilpotent and as $N$ is nilpotent, a well known criterion of P. Hall [2] shows that $G$ is nilpotent.

\subsection{Minimal three generator counterexamples}

It comes hardly as a surprise that the last theorem does not generalise to all solvable groups. Let $G=\left\langle a_{1}, a_{2}, \ldots, a_{r}\right\rangle$ be a solvable group. If any quotient of $G$ that is abelian by nilpotent must be nilpotent a simple inductive argument shows that $G$ must be nilpotent. We therefore focus on groups that are abelian by nilpotent. By another well known result of $\mathrm{P}$. Hall such a group must be residually finite [3]. We are therefore led to considering finite groups that are abelian by nilpotent. We restrict our investigation to three generator groups, as that will suffice for our purposes here. So we consider a group $G=\langle a, b, c\rangle$ that is abelian by nilpotent and that is of type $(r, s, t)$. We are interested in finding criteria for the group to be nilpotent so to begin with we look for minimal counterexamples. We mean here minimal in the sense that no proper quotient is non-nilpotent and that no proper subgroup of type $(r, s, t)$ is non-nilpotent. Suppose then that $G$ is a minimal counterexample. Suppose that the generators $a, b, c$ are $\left\{p_{1}, p_{2}, \ldots, p_{n}\right\}$-elements and that

$$
\begin{aligned}
a & =a_{1} a_{2} \cdots a_{n} \\
b & =b_{1} b_{2} \cdots b_{n} \\
c & =c_{1} c_{2} \cdots c_{n} .
\end{aligned}
$$


are the unique factorisations of $a, b, c$ in the cyclic groups $\langle a\rangle,\langle b\rangle,\langle c\rangle$, where $a_{i}, b_{i}, c_{i}$ are $p_{i}$-elements. If $i \neq j$ then $\left\langle a_{i}, b_{j}\right\rangle=\left\langle a^{l}, b^{m}\right\rangle$ for some integers $l$ and $m$, and thus nilpotent. As the elements $a_{i}, b_{j}$ are coprime it follows that they commute. This argument shows also that $a_{i}, b_{i}$ commute with $c_{j}$ if $i \neq j$. Let $G_{i}=\left\langle a_{i}, b_{i}, c_{i}\right\rangle$. From what we have seen it follows that $G=G_{1} \cdots G_{n}$, a product of normal subgroups, and

$$
\gamma_{m}(G)=\gamma_{m}\left(G_{1}\right) \gamma_{m}\left(G_{2}\right) \cdots \gamma_{m}\left(G_{n}\right) .
$$

As $G$ is non-nilpotent we must have that one of the $G_{i}, i=1, \ldots, n$, is nonnilpotent. By minimality we have then that $G=G_{i}$. So $G$ is generated by $p$-elements for some prime $p$.

Also by minimality, $G$ has a unique minimal normal subgroup $N$ and $G / N$ is a nilpotent group generated by $p$-elements and thus a $p$-group. As $G$ is solvable $N$ is an elementary abelian $q$-group for some prime $q \neq p$. Let $P$ be a Sylow $p$-subgroup of $G$, so $G=P N$. The subgroup $P \cap C_{G}(N)$ is centralised by $N$ and normalised by $P$. As $N$ is the unique minimal normal subgroup it follows that $P \cap C_{G}(N)=1$. This implies that $P$ acts faithfully on $N$ by conjugation and we can think of $P$ as being a subgroup of $\mathrm{GL}(N)$.

Lemma $7 N_{G}(P)=P$.

Proof Otherwise there is some $x \in N$ that normalises $P$. Then for any $y \in P$ we have $[y, x] \in P \cap N=1$ and $x$ centralises $P$. It follows that $x \in Z(G)$ and as $N$ was the minimal normal subgroup we must have that $N=\langle x\rangle$ and $G$ is nilpotent. By this contradiction it is clear that $N_{G}(P)=P$.

It follows that there are exactly $\left[G: N_{G}(P)\right]=|N|$ Sylow $p$-subgroups, namely

$$
\left\{P^{u}: u \in N\right\} \text {. }
$$

In particular if $N$ has dimension $n$ then $q^{n} \equiv 1 \quad(\bmod p)$.

Lemma 8 If $f=e^{x}$ with $e, f \in P$ and $x \in N$ then $e=f$ and $x$ commutes with e.

Proof We have that $e, e^{x} \in P$ and thus

$$
e^{-1} e^{x}=[e, x] \in P \cap N=1 .
$$

Thus $x$ commutes with $e$ and $f=e^{x}=e$. 
Lemma 9 Suppose that $G=\langle a, b, c\rangle$ where $a, b$ and $c$ are $p$-elements as before. There exist linearly independent elements $x, y \in N$ such that

$$
\begin{aligned}
a^{x} & =a, \\
b^{y} & =b, \\
c^{x y} & =c .
\end{aligned}
$$

Proof Let $P$ be a Sylow $p$-subgroup containing $a$ and $c$. Let $x \in N$ such that $a, b \in P^{x}$. As $a \in P \cap P^{x}$ we know from last lemma that $a$ commutes with $x$. If $b=d^{x}$ with $d \in P$ then

$$
G=\left\langle a, d^{x}, c\right\rangle=\left\langle a^{x}, d^{x}, c\right\rangle .
$$

Let $y \in N$ such that $\left\langle d, c^{x^{-1}}\right\rangle=\langle b, c\rangle^{x^{-1}} \leq P^{y}$. Then $d \in P \cap P^{y}$ and $d$ commutes with $y$ and then $b=d^{x}$ commutes also with $y$. Then as $c^{x^{-1}} \in$ $P^{x^{-1}} \cap P^{y}$ we have that $c \in P \cap P^{x y}$ and $c$ commutes with $x y$. Thus

$$
G=\left\langle a, d^{x}, c\right\rangle=\left\langle a^{x}, d^{x}, c\right\rangle=\left\langle a, d^{x y}, c^{x y}\right\rangle .
$$

Finally $x$ and $y$ must be linearly independent since otherwise $a$ commutes with $y$ and $G=\left\langle a^{x y}, d^{x y}, c^{x y}\right\rangle$ would be nilpotent.

Lemma 10 If $G$ is a minimal counterexample that is abelian-by-(class $c$ ) then $\gamma_{c}(G)=N$. In particular, it follows that $P$ is nilpotent of class $c$.

Proof. We have that $\gamma_{c}(G)$ is abelian. Let $R$ be the Sylow-p subgroup of $\gamma_{c}(G)$. As $N$ is the unique minimal normal subgroup of $G, R$ must be trivial. Hence $\gamma_{c}(G) \leq N$ and as $G$ is non-nilpotent and again as $N$ is the unique minimal normal subgroup it follows that $\gamma_{c}(G)=N$. In particular $\gamma_{c}(P) \leq N$ and therefore trivial.

If $N$ has rank $n$, we have seen that $p$ divides $q^{n}-1$. Suppose $q$ has order $r$ modulo $p$ and let $F$ be the Galois field of order $q^{r}$. Then we can think of $N$ as a vector space over $F$ of dimension $n / r$. Suppose that $F$ has basis $e_{1}, \ldots, e_{r}$ over the field of $q$ elements, where $e_{1}=1$. Let $d=n / r$. If

$$
N=\underbrace{F \oplus F \oplus \cdots \oplus F}_{d}
$$


we let $v_{i}$ be the vector $\left(\delta_{1 i}, \ldots, \delta_{d i}\right)$ and for $0 \leq i \leq d-1$ and $1 \leq j \leq r$ we let $u_{i r+j}=e_{j} v_{i}$. Hence $u_{1}, \ldots, u_{n}$ is a basis for $N$ over the field of $q$ elements and $v_{1}=u_{1}, v_{2}=u_{1+r}, \ldots, v_{d}=u_{1+(d-1) r}$ is a basis for $N$ over $F$. Notice that if $p=2$ then $r=1$ and $F$ is the field of $q$ elements. In this manner we have that $\mathrm{GL}_{n / r}(F)$ embeds into $\mathrm{GL}_{n}(q)$. Let $D$ be the diagonal subgroup of $\mathrm{GL}_{n / r}(F)$ with respect to the basis $\left\{u_{1}, u_{r+1}, u_{2 r+1} \cdots, u_{(d-1) r+1}\right\}$. For each $d$-tuple $\left(\lambda_{1}, \ldots, \lambda_{d}\right) \in F^{d}$ we get an element in $D$ that maps $f_{1} v_{1}+\cdots+f_{d} v_{d}$ to $\lambda_{1} f_{1} v_{1}+\cdots+\lambda_{d} f_{d} v_{d}$. We also have that any element $\sigma \in S_{d}$ acts naturally on $N$ by mapping $f_{1} v_{1}+\cdots+f_{d} v_{d}$ to $f_{\sigma(1)} v_{\sigma(1)}+\cdots+f_{\sigma(d)} v_{\sigma(d)}$. Let $D(p)$ be the Sylow $p$-subgroup of $D$ and $S_{d}(p)$ be a Sylow $p$-subgroup of $S_{d}$.

Lemma 11 If $p$ is an odd prime, then the semidirect product $D(p) S_{d}(p)$ is a Sylow p-subgroup of $\mathrm{GL}(n, q)$.

Proof We have that

$$
\left|\mathrm{GL}_{n}(q)\right|=\left(q^{n}-1\right) \cdot\left(q^{n}-q\right) \cdots\left(q^{n}-q^{n-1}\right) .
$$

We know that the order of $q$ modulo $p$ is $r$. Thus $p$ divides $q^{n}-q^{i}$ if and only if $r \mid i$. The highest $p$-power factor of $\left|\mathrm{GL}_{n}(q)\right|$ thus divides

$$
\left(q^{n}-1\right)\left(q^{n}-q^{r}\right)\left(q^{n}-q^{2 r}\right) \cdots\left(q^{n}-q^{(d-1) r}\right),
$$

which is equal to

$$
\left(q^{r}\right)^{d(d-1) / 2} \cdot\left(q^{r}-1\right)^{d} \cdot\left(q^{r}+1\right)\left(q^{2 r}+q^{r}+1\right) \cdots\left(q^{(d-1) r}+q^{(d-2) r}+\cdots+1\right) .
$$

Clearly the highest $p$-power factor of $\left(q^{r}-1\right)^{d}$ is the order of $D(p)$. So it suffices to show that

$$
\left(q^{r}+1\right)\left(q^{2 r}+q^{r}+1\right) \cdots\left(q^{(d-1) r}+q^{(d-2) r}+\cdots+1\right)
$$

has the same highest $p$-power factor as $d$ !. As $q^{r} \equiv 1 \quad(\bmod p)$, we have that $q^{(m-1) r}+\cdots+1$ is divisible by $p$ if and only if $p \mid m$. We finish the proof by showing that $m$ and $q^{(m-1) r}+\cdots+1$ have the same highest $p$-factor. Suppose $q^{r}=1+x p$ and that $m=p^{i} y$ where $y$ is coprime to $p$. We need to show that

$$
\sum_{k=0}^{p^{i} y-1}(1+p x)^{k} \equiv p^{i} y \quad\left(\bmod p^{i+1}\right) .
$$


To see this notice that the left hand side is equal to

$$
p^{i} y+\left(\begin{array}{c}
p^{i} y \\
2
\end{array}\right) p x+\left(\begin{array}{c}
p^{i} y \\
3
\end{array}\right)(p x)^{2}+\cdots\left(\begin{array}{c}
p^{i} y \\
p^{i} y
\end{array}\right)(p x)^{p^{i} y-1} .
$$

We want to see that apart from the first term all the other terms are divisible by $p^{i+1}$. Thus consider

$$
\left(\begin{array}{l}
p^{i} y \\
p^{j} z
\end{array}\right)(p x)^{p^{j} z-1}, \text { with }(z, p)=1
$$

where $2 \leq p^{j} z \leq p^{i} y$. This is equal to $p^{i-j+p^{j} z-1}$. When $j=0$ then $z \geq 2$ and thus $i-j+p^{j} z-1=i+z-1 \geq i+1$. We are left with the case when $j \geq 1$. But then $i-j+p^{j}-1 \geq i-j+(1+2)^{j}-1 \geq i-j+1+j \cdot 2+2^{j}-1 \geq i+1$.

When $p$ is an odd prime we can assume that $P$ is a subgroup of the Sylowsubgroup from last lemma. Let $\lambda$ be any element in $F$ of $p$-power order. Then the multiplication by $\lambda$ on $N=F^{d}$ induces a linear map on $N$. Clearly this linear map commutes with everything in $D(p)$ and $S_{d}(p)$ which implies that any element in $P$ induces a $F$-linear map on $N$. We will need this in next section.

\subsection{Groups that are abelian-by-(class 2)}

Let $G=\langle a, b, c\rangle$ be of type $(r, s, t)$ and suppose that $\gamma_{3}(G)$ is abelian. We want to find criteria under which $G$ must be nilpotent. Let $G=P N$ therefore be a finite minimal counterexample as described in last section. Here we will use additive notation for the group operation in $N$. By Lemma 10 we know that $P$ is nilpotent of class 2 . Let $a, d, c, x, y$ be as in previous section. Thus $b=d^{x}$,

$$
P=\langle a, d, c\rangle, \quad x^{a}=x, \quad y^{d}=y, \quad(x+y)^{c}=x+y,
$$

and

$$
G=\left\langle a, d^{x}, c\right\rangle=\left\langle a^{x}, d^{x}, c\right\rangle=\left\langle a, d^{x+y}, c^{x+y}\right\rangle .
$$

Lemma 12 With notation as above, we have $a^{p}=d^{p}=c^{p}=[a, d]^{p}=$ $[c, d]^{p}=[a, c]^{p}=1$.

Proof We first show that $[a, d]^{p}=1$. By minimality

$$
H=\left\langle a, b^{p}, c\right\rangle=\left\langle a, d^{p x}, c\right\rangle
$$


is nilpotent. Let $P^{z}, z \in N$, be a Sylow- $p$ subgroup that contains $H$. As $a, c, d^{p x} \in P \cap P^{z}$ it follows from Lemma 8 that $a^{z}=a, c^{z}=c$ and $d^{p(z-x)}=d^{p}$. As $c^{x} \neq c$ we must have that $z-x \neq 0$ and as $N$ is irreducible we have that $G=\langle a, d, c, z-x\rangle$. Then both $a$ and $d^{p}$ commute with $z-x$ and thus $[a, d]^{p}$ is in $Z(G)$. As $N$ is the unique minimal normal subgroup of $G$ it follows that $[a, d]^{p}=1$. Similar argument shows that $[a, c]^{p}=[c, d]^{p}=1$. From the reasoning above it follows that $a^{p}$ commutes with $x, a, b, c$ and is therefore in $Z(G)$. Again, as $N$ is the unique minimal normal subgroup of $G$ is follows that $a^{p}=1$. Similarly $d^{p}=c^{p}=1$.

We have now enough material to find all the minimal counterexamples that are abelian-by-(class 2). For the time being we will assume that the prime $p$ is odd in order to be able to apply Lemma 11. We will turn back to the prime 2 later. Notice first that none of $a, d, c$ can be in $Z(P)$. To see this we argue by contradiction and without loss of generality we can assume that $a \in Z(P)$. Then $a$ commutes with $a, d, c$ and $x$ that generate $G$. This contradicts the fact that $N$ is the unique minimal normal subgroup of $G$. It follows that at most one of the commutators $[a, d],[d, c]$ and $[c, a]$ can be trivial. We deal first with the case when exactly one of these is trivial. Without loss of generality we can suppose that $[a, d]=1$. As $[c, a]$ is of order $p$ and in $Z(P)$ and as $N$ is an irreducible $F P$-module, it follows that $[c, a]$ acts like scalar multiplication by some $\lambda$ in $F$ of order $p$ in $F \backslash\{1\}$. Similarly $[d, c]$ acts like multiplication by a scalar and by replacing $d$ by some $d^{i}$ if necessary we can assume that $[c, d]=[c, a]$. Let $z$ be a non-trivial element of $N$ that is fixed by $c$. As $N$ is an irreducible $F P$-module, it follows that $N$ is generated as an FP-module by

$$
z^{a^{i} d^{j}}, \quad 0 \leq i, j \leq p-1 .
$$

As $a$ and $d$ commute we have that the subspace of $N$ fixed by $a$ is $d$-invariant. And as $d \neq 1$ is of order $p$ and $p$ divides $|F|-1$, it follows that there exists an element in $u \in N$ that is fixed by $a$ and such that $u^{d}=\lambda^{j} u$ for some $1 \leq j \leq p-1$. As $N$ is irreducible, it follows that $N$ is generated as an $F P$-module by

$$
u, u^{c}, \ldots, u^{c^{p-1}}
$$

Calculations show that

$$
\begin{aligned}
& u^{c^{i} a}=\lambda^{i} u^{c^{i}}, \\
& u^{c^{i} b}=\lambda^{i+j} u^{c^{i}} .
\end{aligned}
$$


In particular $u, u^{c}, \ldots, u^{c^{p-1}}$ are eigenvectors with respect to distinct eigenvalues for $a$ and they are therefore linearly independent and form a basis for $N$ as a vector space over $F$. From the equations above we must have

$$
\begin{aligned}
& x=\alpha u, \\
& y=\beta u^{c^{-j}} .
\end{aligned}
$$

for some non-trivial $\alpha, \beta \in F$. As $x$ and $y$ are linearly independent we must have that $j$ is coprime to $p$. We have been restricting to odd primes. The fact that $x+y$ must be fixed by $c$ shows that no minimal counterexample exists in that case. We are thus left only with $p=2$ are a possibility. The calculations above suggest the following example:

Example 1. Let $N$ be a vector space of dimension two over the field of $q$-elements where $q \neq 2$. Suppose that with respect to some fixed basis

$$
a=\left[\begin{array}{rr}
1 & 0 \\
0 & -1
\end{array}\right], \quad c=\left[\begin{array}{ll}
0 & 1 \\
1 & 0
\end{array}\right], \quad d=a^{c}=\left[\begin{array}{rr}
-1 & 0 \\
0 & 1
\end{array}\right] .
$$

Let $x=(1,0)$. One can easily check that $P$ is a 2 -group of order 8 . Let $G$ be the semidirect of $N$ by $P=\langle a, d, c\rangle$. One can verify that $G=\left\langle a, d^{x}, c\right\rangle$ is a non-nilpotent $(1,2,2)$-group that is abelian-by-(class 2$)$. It is clearly a minimal counter example.

Assuming that one of $[a, d],[d, c],[c, a]$ was non-trivial therefore led us to a family of minimal counterexamples where in all the examples $P$ is of order 8. We can thus assume from now on that all of $[a, d],[d, c]$ and $[c, a]$ are non-trivial. As we have seen before these are all multiplications by a scalar of order $p$. Replacing $a, d, c$ by some $a^{i}, d^{j}, c^{k}$ we can assume that

$$
[d, a]=[c, d]=[a, c]=\lambda \text {. }
$$

Calculations show that $a d c$ is then in $Z(P)$. As we have already counterexamples when $p=2$, we will assume that $p \geq 3$. Here $a d c$ is of order $p$ and therefore a power of $[a, d]$. Hence $P=\langle a, d\rangle$ and $N$ is generated as a vector space over $F$ by

$$
x, x^{d}, \ldots, x^{d^{p-1}} .
$$

As before we see that

$$
x^{d^{i} a}=\lambda^{i} x^{d^{i}}
$$


and $x, x^{d}, \ldots, x^{d^{p-1}}$ are eigenvectors for $a$ with respect to distinct eigenvalues. Hence these vectors form a basis for $N$ over $F$. Now as $y$ is fixed by $d$ we must have

$$
y=e\left(x+x^{d}+\ldots+x^{d^{p-1}}\right)
$$

for some non-trivial $e \in F$. As $(a d c)^{-1}=c^{-1} d^{-1} a^{-1}$ is in $Z(P)$ and of order $p$ we must have that it is equal to some power of $[d, a]$. Hence

$$
c^{-1}=a d[d, a]^{r}
$$

for some $0 \leq r \leq p-1$. As $x+y$ is fixed by $c$ it follows that

$$
\left((e+1) x+e x^{d}+e x^{d^{2}}+\cdots+e x^{d^{p-1}}\right)^{a d[d, a]^{r}}=(e+1) x+e x^{d}+e x^{d^{2}}+\cdots+e x^{d^{p-1}} .
$$

From these we get the following equations

$$
\begin{aligned}
e+1 & =e \cdot \lambda^{r-1} \\
e & =(e+1) \cdot \lambda^{r} \\
e & =e \lambda^{r+1} \\
& \vdots \\
e & =e \lambda^{r+p-2} .
\end{aligned}
$$

As $\lambda \neq 1$ we must have $p=3$. It follows then from the equations that $r=-1$ and $e=1 /(\lambda-1)$. So when $p \geq 3$ then $p$ must be three and the situation is as in the following example:

Example 2. Let $q$ be a prime distinct from 3 and suppose that the order of $q$ modulo 3 is $r$. Let $F$ be the field of $q^{r}$ elements and let $\lambda$ be an element in $F \backslash\{0\}$ of order 3 . Let $N$ be a three dimensional vectors space over $F$ and with respect to some fixed basis let

$$
a=\left[\begin{array}{rrr}
1 & 0 & 0 \\
0 & \lambda & 0 \\
0 & 0 & \lambda^{2}
\end{array}\right], \quad d=\left[\begin{array}{lll}
0 & 0 & 1 \\
1 & 0 & 0 \\
0 & 1 & 0
\end{array}\right], \quad c=a d[a, d]=\left[\begin{array}{rrr}
0 & 0 & \lambda \\
\lambda^{2} & 0 & 0 \\
0 & 1 & 0
\end{array}\right]
$$

Then $P=\langle a, d\rangle$ is a 3 -group of order 27 and class 2 . Let $x=(1,0,0)$, $y=\frac{1}{\lambda-1}(1,1,1)$. Then $a$ fixes $x, b$ fixes $y$ and $c$ fixes $x+y$. Furthermore $G=\left\langle a, b^{x}, c\right\rangle$ is non-nilpotent and abelian-by-(class 2). In fact $G$ is the semidirect product of $N$ by $P$. 
Proposition 1 Let $G=\left\langle a_{1}, \ldots, a_{r}\right\rangle$ be a group that is abelian-by-(class 2 ) and for which every pair of generators generates a nilpotent group. If none of the generators has order that is divisible by 2 or 3 then $G$ is nilpotent.

Proof As $G$ is abelian by nilpotent we know that $G$ is residually finite and we can assume that $G$ is finite. We argue by contradiction and suppose that we have a minimal counterexample. A similar argument as we used for three generator groups shows that $G$ is an extension of a elementary abelian $q$-group $N$ by a $p$-group $P$ of class at most 2 . We know also that we can assume that all the generators are $p$-elements. Therefore $\left[a_{i}, a_{j}, a_{k}\right] \in N$ for all $1 \leq i, j, k \leq r$. We want to show that all such commutators must be trivial. If not we get a non-nilpotent group $\left\langle a_{i}, a_{j}, a_{k}\right\rangle$ where $a_{i}, a_{j}, a_{k}$ are $p$-elements for some $p \neq 2,3$. But we have seen that no such group exists.

As we said in the introduction we conjecture that the proposition above extends to groups that are abelian-by-(class $c)$ and that there is a finite set $\mathcal{P}_{c}$ of exceptional primes such that for any pairwise nilpotent presentation of a abelian-by-( $\operatorname{class} c)$ group is nilpotent if the generators are of $P_{c}$-free order. The following example gives some information for how big the exceptional primes must be.

Example 3. Take an odd prime $p$ and any other prime $q$. Suppose that $q^{n} \equiv 1$ modulo $p$ and that $K$ is the Galois field of order $q^{n}$. Let $\lambda$ be an element in $K$ that generates the cyclic Sylow $p$-subgroup of $K \backslash\{0\}$. Let $N$ be a vector space over $K$ of dimension $p$ with basis $u_{0}, u_{1}, \ldots, u_{p-1}$. For $i=0, \ldots, p-1$ let $d_{i}$ be the linear map that maps $u_{i}$ to $\lambda u_{i}$ but fixes the other basis vectors. Let $\sigma$ be the linear map that cyclically permutes $u_{0}, \ldots, u_{p-1}$. Let $r=(p-3) / 2$ and

$$
\begin{aligned}
a & =\left[d_{0, r} \sigma\right]=d_{0}^{(-1+\sigma)^{r}}, \\
b & =a^{\sigma^{p-1-r}}, \\
c & =\sigma .
\end{aligned}
$$

As $2(p-1-r)=2(p-1)-(p-3)=p+1>p$ we can then choose linearly independent vectors $x, y$ such that $x+y=u_{0}+u_{1}+\cdots+u_{p-1}$ where $a$ fixes $x, b$ fixes $y$ and $c$ fixes $x+y$. For example we can take $x=(\underbrace{0, \cdots, 0}_{(p+1) / 2}, \underbrace{1, \cdots, 1}_{(p-1) / 2})$ and $y=(\underbrace{1, \cdots, 1}_{(p+1) / 2}, \underbrace{0, \cdots, 0}_{(p-1) / 2})$. As $\left\langle d_{0}, \sigma\right\rangle$ is nilpotent of class $p$ we have that 
$P=\langle a, c\rangle$ is nilpotent of class $p-r=(p+3) / 2$. Consider now the group

$$
G=\left\langle a, b^{x}, c\right\rangle .
$$

Then $G$ is nilpotent-by-(class $s)$ where $s=(p+3) / 2$ and $G$ is also of type $(1, s, s)$. To see that $G$ is non-nilpotent one can calculate directly that $\left[b^{x}, c, a\right]$ is a non-trivial element in $N$. The prime $p$ is equal to $2 s-3$. This shows that if there is a largest exceptional prime, then it is at least equal to $2 s-3$.

\section{Bounds for the nilpotency class}

In this last section, we investigate the possibility to bound the nilpotency class of a finite $p$-group $G=\langle a, b, c\rangle$ of type $(r, s, t)$. First we deal with cases where such a bound exists.

\subsection{Some positive results}

Let $G=\langle a, b, c\rangle$ be a three generator pairwise nilpotent group. We say that $G$ is of type $(r, s,-)$ if the exact value of $t$ is immaterial.

Frequently we shall use the following relations, valid in any group $G$ :

$$
\begin{gathered}
{[x, y, z] \equiv[x, z, y] \cdot[x,[y, z]] \quad\left(\bmod \gamma_{4}(G)\right)} \\
\text { (or equivalently }[x,[y, z]] \equiv[x, y, z] \cdot[x, z, y]^{-1} \quad\left(\bmod \gamma_{4}(G)\right)
\end{gathered}
$$

and

$$
\left[x^{-1}, y\right] \equiv\left[x, y^{-1}\right] \equiv[x, y]^{-1} \equiv[y, x] \quad\left(\bmod \gamma_{3}(G)\right) .
$$

Lemma 13 In a group $G=\langle a, b, c\rangle$ of type $(1,2,-)$, we have:

(i) $[x, y, z]=1$ when $x, y, z \in\{a, b\}$ or $x, y, z \in\{a, c\}$ (and also when $x, y, z \in\{b, c\}$ if $G$ is of type $(1,2,2))$;

(ii) $[a, b, c]=[b, a, c]=1$;

(iii) $[a, c, b] \equiv[c, a, b]^{-1} \equiv[c, b, a]^{-1} \equiv[b, c, a] \quad\left(\bmod \gamma_{4}(G)\right)$. 
Proof Easy (in particular, (i) and (ii) are obvious).

Lemma 14 In a group $G=\langle a, b, c\rangle$ of type $(1,2,-)$, we have modulo $\gamma_{5}(G)$ :

(i) $[a, c, b, a] \equiv 1$;

(ii) $[c, a,[b, c]] \equiv[a, c, b, c]^{-1}$;

(iii) $[b, c, c, a] \equiv[a, c, b, c]^{2}$;

(iv) $[b, c, b, a] \equiv[a, c, b, b]$.

Proof $(\mathrm{i})[a, c, b, a] \equiv[a, c, a, b] \cdot[a, c,[b, a]] \equiv 1 \quad\left(\bmod \gamma_{5}(G)\right)$ since $[a, c, a]=$ $[b, a]=1$.

(ii) $[c, a,[b, c]] \equiv[c, a, b, c] \cdot[c, a, c, b]^{-1} \equiv[c, a, b, c] \equiv[a, c, b, c]^{-1} \quad\left(\bmod \gamma_{5}(G)\right)$.

(iii) We have $[b, c, c, a] \equiv[b, c, a, c] \cdot[b, c,[c, a]] \quad\left(\bmod \gamma_{5}(G)\right)$, hence

$$
[b, c, c, a] \equiv[a, c, b, c] \cdot[b, c,[c, a]] \quad\left(\bmod \gamma_{5}(G)\right)
$$

for $[b, c, a] \equiv[a, c, b] \quad\left(\bmod \gamma_{4}(G)\right)$. Since $[b, c,[c, a]]=[c, a,[b, c]]^{-1}$, the result follows from (ii).

(iv) $[b, c, b, a] \equiv[b, c, a, b] \cdot[b, c,[b, a]] \quad\left(\bmod \gamma_{5}(G)\right)$. Since $[b, a]=1$ and $[b, c, a] \equiv[a, c, b] \quad\left(\bmod \gamma_{4}(G)\right)$, we obtain the desired result.

Proposition 2 For any prime $p \geq 3$, the nilpotency class of a finite $p$-group $G=\langle a, b, c\rangle$ of type $(1,2,2)$ is at most 3 .

Proof We must show that $\left[x_{1}, x_{2}, x_{3}, x_{4}\right] \equiv 1 \quad\left(\bmod \gamma_{5}(G)\right)$ for all $x_{i} \in$ $\{a, b, c\}$. Actually, by Lemma 13 , it suffices to verify that $[a, c, b, x] \equiv 1$ $\left(\bmod \gamma_{5}(G)\right)$ for $x=a, b$ or $c$.

Case 1: $x=a$. This is Lemma 14(i).

Case 2: $x=b$. By Lemma 13(iii), $[a, c, b, b] \equiv[b, c, a, b] \quad\left(\bmod \gamma_{5}(G)\right)$, and

$$
[b, c, a, b] \equiv[b, c, b, a] \cdot[b, c,[a, b]] \equiv 1 \quad\left(\bmod \gamma_{5}(G)\right)
$$

for $[b, c, b]=[a, b]=1$.

Case 3: $x=c$. By Lemma 14(iii), $[a, c, b, c]^{2} \equiv[b, c, c, a] \equiv 1 \quad\left(\bmod \gamma_{5}(G)\right)$ since $[b, c, c]=1$. But $p$ is odd, so $[a, c, b, c] \equiv 1 \quad\left(\bmod \gamma_{5}(G)\right)$, as required. 
Lemma 15 In a group $G=\langle a, b, c\rangle$ of type $(1,2,3)$, we have modulo $\gamma_{6}(G)$ :

(i) $[a, c, b, b, a] \equiv 1$;

(ii) $[a, c, b, b, b] \equiv 1$;

(iii) $[a, c, b, c, b]^{2} \equiv 1$;

(iv) $[a, c, b, c, c]^{3} \equiv 1$.

Proof (i) We can write

$$
[a, c, b, b, a] \equiv[a, c, b, a, b] \cdot[a, c, b,[b, a]] \equiv[a, c, b, a, b] \quad\left(\bmod \gamma_{6}(G)\right),
$$

and by Lemma 14(i), this last commutator belongs to $\gamma_{6}(G)$.

(ii) By Lemma 13(iii), $[a, c, b, b, b] \equiv[b, c, a, b, b] \quad\left(\bmod \gamma_{6}(G)\right)$. Since $a$ and $b$ commute and $[b, c, b, b]=1$, we can write

$$
[b, c, a, b, b] \equiv[b, c, b, a, b] \equiv[b, c, b, b, a] \equiv 1 \quad\left(\bmod \gamma_{6}(G)\right)
$$

and the result follows.

(iii) We have $[a, c, b, c, b]^{2} \equiv\left[[a, c, b, c]^{2}, b\right] \quad\left(\bmod \gamma_{6}(G)\right)$ and so, by Lemma 14(iii), $[a, c, b, c, b]^{2} \equiv[b, c, c, a, b] \quad\left(\bmod \gamma_{6}(G)\right)$. As in the preceding case, since $a$ and $b$ commute and $[b, c, c, b]=1$, we have

$$
[b, c, c, a, b] \equiv[b, c, c, b, a] \equiv 1 \quad\left(\bmod \gamma_{6}(G)\right)
$$

and the result follows.

(iv) Using once again Lemma 14(iii), we can write

$$
[a, c, b, c, c]^{2} \equiv\left[[a, c, b, c]^{2}, c\right] \equiv[b, c, c, a, c] \quad\left(\bmod \gamma_{6}(G)\right) .
$$

It remains to verify that $[b, c, c, a, c] \equiv[a, c, b, c, c]^{-1} \quad\left(\bmod \gamma_{6}(G)\right)$. For that, we can write modulo $\gamma_{6}(G)$ :

$$
\begin{aligned}
& {[b, c, c, a, c] \equiv[b, c, c, c, a] \cdot[b, c, c,[a, c]] \equiv[b, c, c,[a, c]]} \\
& \equiv[b, c,[a, c], c] \cdot[b, c,[c,[a, c]]] \equiv[b, c,[a, c], c] \\
& \equiv[b,[a, c], c, c] \cdot[b,[c,[a, c]], c] \equiv[b,[a, c], c, c] \\
& \equiv \quad\left[[a, c, b]^{-1}, c, c\right] \quad \equiv[a, c, b, c, c]^{-1}
\end{aligned}
$$

and the proof is complete. 
Lemma 16 In a group $G=\langle a, b, c\rangle$ of type $(1,2,-)$, we have

$$
[a, c, b, b, c, a] \equiv[a, c, b, c, a, b] \quad\left(\bmod \gamma_{7}(G)\right)
$$

Proof First notice that

$$
[a, c, b, b, c, a] \equiv[a, c, b, b, a, c] \cdot[a, c, b, b,[c, a]] \quad\left(\bmod \gamma_{7}(G)\right)
$$

In this product, consider the first factor: since $a$ and $b$ commute, we have

$$
[a, c, b, b, a, c] \equiv[a, c, b, a, b, c] \equiv[a, c, a, b, b, c] \equiv 1 \quad\left(\bmod \gamma_{7}(G)\right) .
$$

Now consider the second factor:

$$
[a, c, b, b,[c, a]] \equiv[a, c, b,[c, a], b] \cdot[a, c, b,[b,[c, a]]] \quad\left(\bmod \gamma_{7}(G)\right)
$$

Since $[a, c, b] \equiv[b,[c, a]] \quad\left(\bmod \gamma_{4}(G)\right)$, we have $[a, c, b,[b,[c, a]]] \equiv 1 \quad\left(\bmod \gamma_{7}(G)\right)$. Therefore, we have

$$
[a, c, b, b, c, a] \equiv[a, c, b,[c, a], b] \quad\left(\bmod \gamma_{7}(G)\right)
$$

Since $[a, c, b] \equiv[b, c, a] \quad\left(\bmod \gamma_{4}(G)\right)$ by Lemma $13($ iii $)$, we can write

$$
\begin{aligned}
{[a, c, b, b, c, a] } & \equiv[b, c, a,[c, a], b] \\
& \equiv[b, c,[c, a], a, b] \cdot[b, c,[a,[c, a]], b] \\
& \equiv[b, c,[c, a], a, b] \\
& \equiv[b,[c, a], c, a, b] \cdot[b,[c,[c, a]], a, b] \\
& \equiv[b,[c, a], c, a, b] \\
& \equiv[a, c, b, c, a, b]
\end{aligned}
$$

as required.

Lemma 17 In a group $G=\langle a, b, c\rangle$ of type $(1,2,3)$, we have modulo $\gamma_{7}(G)$ :

(i) $[a, c, b, c, a, a] \equiv 1$;

(ii) $[a, c, b, c, a, b]^{2} \equiv 1$;

(iii) $[a, c, b, c, a, c]^{6} \equiv 1$;

(iv) $[a, c, b, b, c, a]^{2} \equiv 1$; 
(v) $[a, c, b, b, c, b]^{6} \equiv 1$;

(vi) $[a, c, b, b, c, c] \equiv 1$.

Proof (i) We have $[a, c, b, c, a, a] \equiv[a, c, b, a, c, a] \cdot[a, c, b,[c, a], a] \quad\left(\bmod \gamma_{7}(G)\right)$, hence $[a, c, b, c, a, a] \equiv[a, c, b,[c, a], a] \quad\left(\bmod \gamma_{7}(G)\right)$ by Lemma 14(i). Since

$$
[a, c, b,[c, a], a] \equiv[a, c, b, a,[c, a]] \cdot[a, c, b,[[c, a], a]] \equiv 1 \quad\left(\bmod \gamma_{7}(G)\right)
$$

(by applying once again Lemma 14(i)), we obtain the desired result.

(ii) Since $a$ and $b$ commute, we can write

$$
[a, c, b, c, a, b]^{2} \equiv[a, c, b, c, b, a]^{2} \equiv\left[[a, c, b, c, b]^{2}, a\right] \quad\left(\bmod \gamma_{7}(G)\right),
$$

and we are done thanks to Lemma 15(iii).

(iii) We can write modulo $\gamma_{7}(G)$ :

$$
\begin{aligned}
& {[a, c, b, c, a, c] \equiv[a, c, c, b, a, c] \cdot[a, c,[b, c], a, c] \equiv[a, c,[b, c], a, c]} \\
& \equiv[a, c, a,[b, c], c] \cdot[a, c,[b, c, a], c] \equiv \quad[a, c,[b, c, a], c] \\
& \equiv[a, c, c,[b, c, a]] \cdot[a, c,[b, c, a, c]] \equiv \quad[a, c,[b, c, a, c]] \\
& \equiv \quad[[b, c, a, c],[a, c]]^{-1} \quad \equiv[b, c, a, c, a, c]^{-1}[b, c, a, c, c, a] \\
& \equiv[a, c, b, c, a, c]^{-1}[a, c, b, c, c, a] \text {. }
\end{aligned}
$$

It follows that $[a, c, b, c, a, c]^{2} \equiv[a, c, b, c, c, a] \quad\left(\bmod \gamma_{7}(G)\right)$ and so

$$
[a, c, b, c, a, c]^{6} \equiv[a, c, b, c, c, a]^{3} \equiv\left[[a, c, b, c, c]^{3}, a\right] \quad\left(\bmod \gamma_{7}(G)\right) .
$$

Therefore, by Lemma 15(iv), we can say that the element $[a, c, b, c, a, c]^{6}$ belongs to $\gamma_{7}(G)$, as required.

(iv) That follows from (ii) and Lemma 16.

(v) We can write modulo $\gamma_{7}(G)$ :

$$
\begin{aligned}
{[a, c, b, b, c, b] } & \equiv[b, c, a, b, c, b] \\
& \equiv[b, c, b, a, c, b] \\
& \equiv[b, c, b, c, a, b] \cdot[b, c, b,[a, c], b] \\
& \equiv[b, c, b,[a, c], b]
\end{aligned}
$$




$$
\begin{aligned}
& \equiv[b, c, b, b,[a, c]] \cdot[b, c, b,[a, c, b]] \\
& \equiv[a, c, b,[b, c, b]]^{-1} \\
& \equiv[a, c, b,[b, c], b]^{-1}[a, c, b, b,[b, c]] .
\end{aligned}
$$

In this product, we can write the first factor in the form

$$
[a, c, b,[b, c], b]^{-1} \equiv[a, c, b, b, c, b]^{-1}[a, c, b, c, b, b] \quad\left(\bmod \gamma_{7}(G)\right)
$$

and the second one in the form

$$
[a, c, b, b,[b, c]] \equiv[a, c, b, b, b, c][a, c, b, b, c, b]^{-1} \quad\left(\bmod \gamma_{7}(G)\right),
$$

thus $[a, c, b, b,[b, c]] \equiv[a, c, b, b, c, b]^{-1} \quad\left(\bmod \gamma_{7}(G)\right)$ by Lemma $15(\mathrm{ii})$. Consequently, we obtain the relation

$$
[a, c, b, b, c, b] \equiv[a, c, b, b, c, b]^{-1}[a, c, b, c, b, b] \cdot[a, c, b, b, c, b]^{-1} \quad\left(\bmod \gamma_{7}(G)\right)
$$

and it follows that

$$
[a, c, b, b, c, b]^{3} \equiv[a, c, b, c, b, b] \quad\left(\bmod \gamma_{7}(G)\right) .
$$

Thus

$$
[a, c, b, b, c, b]^{6} \equiv[a, c, b, c, b, b]^{2} \equiv\left[[a, c, b, c, b]^{2}, b\right] \quad\left(\bmod \gamma_{7}(G)\right)
$$

and so $[a, c, b, b, c, b]^{6} \equiv 1 \quad\left(\bmod \gamma_{7}(G)\right)$ by Lemma $15(\mathrm{iii})$.

(vi) We have modulo $\gamma_{7}(G)$ :

$$
\begin{aligned}
{[a, c, b, b, c, c] } & \equiv[b, c, a, b, c, c] \\
& \equiv[b, c, b, a, c, c] \\
& \equiv[b, c, b, c, a, c] \cdot[b, c, b,[a, c], c] \\
& \equiv[b, c, b,[a, c], c] \\
& \equiv[b, c, b, c,[a, c]] \cdot[b, c, b,[[a, c, c]] \\
& \equiv 1
\end{aligned}
$$

The proof is now complete. 
Proposition 3 For any prime $p \geq 5$, the nilpotency class of a finite p-group $G=\langle a, b, c\rangle$ of type $(1,2,3)$ is at most 5 .

Proof Using Lemma 13 and Lemma 14(iii,iv), it is easy to see that every commutator of the form $\left[x_{1}, \ldots, x_{6}\right]$ (with $x_{i} \in\{a, b, c\}$ ) is equivalent modulo $\gamma_{7}(G)$ to an expression of the form $[a, c, b, x, y, z]^{\epsilon}$, where $x, y, z \in\{a, b, c\}$ and $\epsilon=0, \pm 1, \pm 2$. Therefore, to prove our proposition, it suffices to show that

$$
[a, c, b, x, y, z] \equiv 1 \quad\left(\bmod \gamma_{7}(G)\right)
$$

for all $x, y, z \in\{a, b, c\}$. For that, consider three cases.

Case 1: $x=a$. The result follows from Lemma 14(i).

Case 2: $x=b$. If $y=a$ or $b$, the result follows from Lemma 15(i,ii); if $y=c$, it is a consequence of Lemma $17(\mathrm{iv}, \mathrm{v}, \mathrm{vi})$.

Case 3: $x=c$. In the same way, the result follows from Lemma 15(iii,iv) if $y=b$ or $c$ and from Lemma 17(i,ii,iii) if $y=a$.

\subsection{Some negative results}

If $R$ is an associative commutative ring with 1 , consider the free associative (non-commutative) $R$-algebra $A$ freely generated by three elements $u, v, w$. Thus $A=\bigoplus_{i \geq 0} A_{i}$, where the elements of $A_{i}$ are linear combinations of monomials of degree $i$.

For positives integers $r, s, t, k$, denote by $I_{k}(r, s, t)$ the ideal of $A$ generated by all elements of the form $x_{1} \ldots x_{n}\left(x_{i} \in\{u, v, w\}\right)$, with one at least of the following conditions holds:

(i) $x_{1}, \ldots, x_{n} \in\{u, v\}$ and $n=r+1$;

(ii) $x_{1}, \ldots, x_{n} \in\{u, w\}$ and $n=s+1$;

(iii) $x_{1}, \ldots, x_{n} \in\{v, w\}$ and $n=t+1$;

(iv) $n=k+1$.

Thanks to the relation $(1+x)\left(1-x+x^{2}-\cdots+(-1)^{k} x^{k}\right)=1+(-1)^{k} x^{k+1}$, it is easy to prove that in the quotient $A / I_{k}(r, s, t)$, the set of cosets of the form $1+y+I_{k}(r, s, t)$ (with $y \in \bigoplus_{i \geq 1} A_{i}$ ) is a multiplicative group, which is 
nilpotent of class at most $k$ (see for example [6, Lemma 9.1]). In this group, consider elements $a, b, c$ defined by

$$
a=1+u+I_{k}(r, s, t), b=1+v+I_{k}(r, s, t), c=1+w+I_{k}(r, s, t)
$$

and denote by $\Gamma_{k}(r, s, t)$ the subgroup generated by these elements. Then $\Gamma_{k}(r, s, t)=\langle a, b, c\rangle$ is of type $(r, s, t)$ : for example, let us verify that the nilpotency class of $\langle a, b\rangle$ is at most $r$. A commutator of the form $\left[x_{1}, \ldots, x_{r+1}\right]$ $\left(x_{i} \in\{a, b\}\right)$ is equal to an element of the form $1+y+I_{k}(r, s, t)$, where $y$ is a linear combination of monomials $y_{1} \ldots y_{n}\left(y_{i} \in\{u, v, w\}\right)$, with $n \geq r+1$ ((see [6, Lemma 9.1]). Since $A$ is free, we can assume that all these $y_{i}$ belong to $\{u, v\}$. It follows that $y$ lies in $I_{k}(r, s, t)$ and so $\left[x_{1}, \ldots, x_{r+1}\right]=1$, as desired. In the sequel, we shall suppose that $R$ is a field of characteristic $p>0$. Then one can see easily that $\Gamma_{k}(r, s, t)$ is a finite $p$-group. The following result shows that the bounds of the nilpotency class obtained in both propositions 2 and 3 are sharp.

Proposition 4 If $R$ is a field of characteristic $p>0$, then:

(i) The nilpotency class of $\Gamma_{3}(1,2,2)$ is 3;

(ii) If $p \neq 2$, the nilpotency class of $\Gamma_{5}(1,2,3)$ is 5 .

Proof First, note that the nilpotency class of $\Gamma_{3}(1,2,2)\left(\operatorname{resp} . \Gamma_{5}(1,2,3)\right)$ is at most 3 (resp. 5).

(i) By $[6$, Lemma 9.1], the commutator $[a, c, b]$ is equal to $1+[u, w, v]+$ $I_{3}(1,2,2)$, where in this latter expression, the brackets must be interpreted as the Lie product. An easy calculation leads to

$$
[u, w, v] \equiv u w v+v w u \quad\left(\bmod I_{3}(1,2,2)\right) .
$$

Clearly, $u w v+v w u$ does not belong to $I_{3}(1,2,2)$. Thus $[a, c, b] \neq 1$ and so $\Gamma_{3}(1,2,2)$ is nilpotent of class 3 exactly.

(ii) Applying [6, Lemma 9.1] again: the commutator $[a, c, b, c, a]$ is equal to $1+[u, w, v, w, u]+I_{5}(1,2,3)$. After a calculation, we get

$$
[u, w, v, w, u] \equiv 2 u w v w u \not \equiv 0 \quad\left(\bmod I_{5}(1,2,3)\right),
$$

hence $[a, c, b, c, a] \neq 1$. 
The results obtained in Propositions 2 and 3 lead to the following question:

Question. Consider positive integers $r, s, t$ (we can assume $r \leq s \leq t$ without loss of generality). Is it possible to find integers $p_{0}$ and $k$ such that, for any prime $p \geq p_{0}$, the nilpotency class of every finite $p$-group of type $(r, s, t)$ is at most $k$ ?

It follows from the next proposition that the answer is negative as from $r=2$ :

Proposition 5 If $R$ is is a field of characteristic $p>0$, the nilpotency class of $\Gamma_{3 k+1}(2,2,2)$ is exactly $3 k+1$ for any integer $k>0$.

Proof Already we know that the nilpotency class of $\Gamma_{3 k+1}(2,2,2)$ is at most $3 k+1$. Thus it suffices to find a non trivial commutator in $a, b, c$ of weight $3 k+1$. By induction on the integer $n>0$, it is easy to prove the relation

$$
[u, v, w, u, \ldots, v, w, u] \equiv u(v w u)^{n}+(-1)^{n} u(w v u)^{n} \quad\left(\bmod I_{3 k+1}(2,2,2)\right),
$$

where the sequence $v, w, u$ appears $n$ times in the Lie product $[u, v, w, u, \ldots, v, w, u]$. Consequently, for $n=k$, we have clearly

$$
[u, v, w, u, \ldots, v, w, u] \not \equiv 0 \quad\left(\bmod I_{3 k+1}(2,2,2)\right) .
$$

By [6, Lemma 9.1], the commutator $[a, b, c, a, \ldots, b, c, a]$ (in which the sequence $b, c, a$ appears $k$ times $)$ is equal to $1+[u, v, w, u, \ldots, v, w, u]+I_{3 k+1}(2,2,2)$ and so is non trivial, as desired.

Therefore, we can restrict to $r=1$. Trivially, there is a positive answer when $s=1$ (for any $t$ ). As we have seen previously (Propositions 2 and 3), we also have a positive answer when $s=2$ and $t=2$ or $t=3$. We conjecture that this answer remains positive for an arbitrary integer $t$ when $r=1$ and $s=2$. On the other hand, the problem has a negative answer when $s \geq 3$ :

Proposition 6 If $R$ is is a field of odd characteristic $p>0$, the nilpotency class of $\Gamma_{4 k+1}(1,3,3)$ is exactly $4 k+1$ for any integer $k>0$.

Proof The nilpotency class of $\Gamma_{4 k+1}(1,3,3)$ is bounded by $4 k+1$. Thanks to an induction on $n$, we can write

$$
[u, w, v, w, u, \ldots, w, v, w, u] \equiv 2^{n} u(w v w u)^{n} \quad\left(\bmod I_{4 k+1}(1,3,3)\right),
$$


where in the Lie product $[u, w, v, w, u, \ldots, w, v, w, u]$ the sequence $w, v, w, u$ appears $n$ times. If $n=k$, we have

$$
[u, w, v, w, u, \ldots, w, v, w, u] \not \equiv 0 \quad\left(\bmod I_{4 k+1}(1,3,3)\right)
$$

since $p \neq 2$. By [6, Lemma 9.1], the commutator $[a, c, b, c, a, \ldots, c, b, c, a]$ (in which the sequence $c, b, c, a$ appears $k$ times) is equal to $1+[u, w, v, w, u, \ldots, w, v, w, u]+$ $I_{4 k+1}(1,3,3)$ and so is non trivial. That completes the proof.

\section{References}

[1] E. S. Golod. Some problems of Burnside type, in: Proc. Int. Congr. Math. (Moscow 1966) (1968), 284-289. English transl.: American Mathematical Society Translations. Series 2, Volume 84: Twelve papers on algebra, algebraic geometry and topology. American Mathematical Society, Providence R. I. 1969.

[2] P. Hall, Some sufficient conditions for a group to be nilpotent, Illinois. J. Math. 2 (1958), 787-801.

[3] P.Hall, On the finiteness of certain soluble groups, Proc. London Math. Soc. 9 (1959), 595-622.

[4] G. Havas and M. R. Vaughan-Lee, 4-Engel groups are locally nilpotent, Int. J. Alg. Com., 15 No. 4 (2005), 649-682.

[5] F. Hermanns, On certain groups of exponent eight generated by three involutions. Burnside groups (Proc. Workshop, Univ. Bielefeld, Bielefield. 1977), 189-210, Lecture Notes in Math., 806, Springer, Berlin (1980).

[6] E. I. Khukhro, p-Automorphisms of Finite p-Groups, London Mathematical Society Lecture Note Series, 246, Cambridge University Press, Cambridge (1998).

[7] G. Traustason, A note on the local nilpotence of 4-Engel groups, Int. J. Alg. Com., 15 No. 4 (2005), 757-764.

[8] M. Zorn, Nilpotency of finite groups, Bull. Amer. Math. Soc. 42 (1936), 485-486. 